\title{
Imaginarios ambientales y de educación ambiental de los estudiantes y docentes de la Institución Educativa Agrotécnico Mixto, municipio de Belén de los Andaquíes (Caquetá)*
}

\author{
Verenice Sánchez Castillo ${ }^{1}$ \\ (D) http://orcid.org/ 0000-0002-4472-6191 \\ Carlos Gómez Cano ${ }^{2}$ \\ (D) http://orcid.org/ 0000-0003-0425-7201 \\ César Coronado Sarria ${ }^{3}$ \\ (D) http://orcid.org/0000-0001-5530-2653 \\ Wilmer Valenzuela Molina ${ }^{4}$ \\ (iD http://orcid.org/0000-0002-2476-4334 \\ Universidad de la Amazonia, Colombia
}

DOI: http://dx.doi.org/10.17081/eduhum.19.32.2537

Recibido: 10 de junio de 2016

Aceptado: 6 de septiembre de 2016

\section{Learning environments involved in environmental education process conducted by students and teachers of the Institucion Educativa Agrotecnico Mixto from Belen de los Andaquies municipality (Caqueta)}

Palabras clave:

Interdisciplinar, Transdisciplinar, Agroecológica

Eđucación ambiental.

Key words:

Interdisciplinary, Transdisciplinary, Agroecological,

Environmental education.

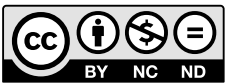

\begin{abstract}
Resumen
El modelo de educación imperante en las instituciones educativas del país presenta graves falencias en cuanto al método de enseñanza de la educación ambiental. Por esto, se busca plantear la transdisciplinariedad entre asignaturas, para que la comunidad educativa se vincule con los diferentes actores que giran en torno a estas, y se pueda evaluar, rediseñar y planificar desde una vista integral en el contexto educativo. En esta dirección, se realizó el presente estudio en la Institución Educativa Agrotécnịco Mixto, lo que implicó, desde el punto de vista metodológico, la ejecución de una investigación cualitativa de estudio de çaso, a partir de entrevistas realizadas a los docentes y estudiantes para conocer sus imaginarios ambientales y de educación ambiental. La producción de los datos permitió identificar divergencias entre ambas percepciones y, a partir de esta realidad, se elaboró una propuesta para el rediseño de percepciones y, a partir de esta realidad, se elaboró una propuesta para el rediseño de como estrategia para los procesos de enseñanza y aprendizaje.
\end{abstract}

\section{Abștract}

Serious shortcomings related to environmental education teaching process are evident in the prevailing model of education inside educational institutions of this country. For this reason, the tranșdisciplinarity between subjects are presented, with the purpose of linking an educational community with the associated actors, and in this way redesign and planned from a holistic view based on educational context, can be evaluated. This study was conducted in Institucion Educativa Agrotecnico Mixto, which means, the implementation of a qualitative case study research from a methodological point of view, through interviews with teachers and students in order to meet their learning environment and environmental educational needs. Through data collection it was possible to identify differences between two point of views and on the basis of this reality, a proposal was suggested to redesign the educational methodology of the institution linking the agro-ecological zones as a strategy for teaching and learning processes.

Referencia de este artículo (APA): Sánchez, V., Gómez, C., Coronado, C. \& Valenzuela, W. (2017). Imaginarios ambientales y de educación ambiental de los estudiantes y docentes de la Institución Educativa Agrotécnico Mixto municipio de Belén de los Andaquíes (Caquetá). En Revista Educación y Humanismo, 19(32), 126-144. http://dx.doi.org/10.17081/eduhum.19.32.2537

* Artículo perteneciente a la línea de Investigación Ambiente y Desarrollo, adscrita al grupo de investigación en Agroecología y Desarrollo Rural. Universidad de la Amazonia Colombia.

1. Ingeniera Agroecóloga. Magíster en Ambiente y Desarrollo. Doctoranda en Antropología. Docente-Investigadora de la Universidad de la Amazonia Colombia. Líder del grupo de investigación en Agroecología y Desarrollo Rural - GIADER. ve.sanchez@udla.edu.co

2. Contador Público. Administrador Público. Especialista en Pedagogía y en Gestión Pública. Maestrando en Ciencias de la Educación, integrante del Grupo de Estudio de Futuro en el Mundo Amazónico - GEMA de la Universidad de la Amazonia, Colombia. carlosgomez325@gmail.com

3. Estudiante del programa de Ingeniería Agroecológica de la Universidad de la Amazonia, Colombia. Integrante del Semillero de Investigación en Ambiente y Desarrollo Rural - SIADER. cyeami2011@hotmail.com

4. Estudiante del programa de Ingeniería Agroecológica de la Universidad de la Amazonia, Colombia. Integrante del Semillero de Investigación en Ambiente y Desarrollo Rural-SIADER. wvm951@gmail.com 


\section{Introducción}

La realidad ambiental es producto de la materialización de nuestros pensamientos $\mathrm{y}$ actitudes en términos de la relación de lo natural y lo humano (Perafán, 2013). Los problemas ambientales son, por tanto, el resultado de dicha relación que es de carácter desigual, ya que, en algunos casos, nos presenta como protectores de la naturaleza y, en otros, como explotadores (Descola \& Palsson, 2001). La solución para los problemas ambientales pareciera hallarse en un manual técnico, cuya simple aplicación conllevaría su solución; sin embargo, la experiencia ha demostrado que esta estrategia para abordar la cuestión ambiental no ha sido tan efectiva.

Una alternativa al respecto se halla en el estudio de los imaginarios, lo que, en primera instancia, busca comprender, a través de los discursos y las actitudes, el comportamiento de las personas y de esta manera construir, luego, opciones de solución negociadas y más próximas con la realidad.

\section{EI concepto de la educación ambiental-EA}

Según Barba y Sanzetenea (2002), la educación es un proyecto humano que se produce como respuesta a contenidos sociales y culturales. Más específicamente, Meseguer, Más, Gil, Hernández y Guilabert (2009) consideran que la educación ambiental es un proceso formativo que busca generar una cultura ambiental mediante el fomento de actitudes, aptitudes, valores, conocimientos y la generación de conciencia, con el fin último del establecimiento de la sustentabilidad.
En una definición más amplia del concepto, Vega y Álvarez (2005) sugieren que la EA no se reduce a educar para "conservar la naturaleza", "concienciar personas" o "cambiar conductas", sino que se imparte con el fin de generar conciencia de las responsabilidades globales y así contribuir en el desarrollo humano.

Rengifo, Quitiaquez y Mora (2012) comparten lo propuesto por Vega y Álvarez, pero además aseguran que los procesos de este tipo deben ser permanentes, tanto en la escuela como fuera de ella, haciendo hincapié en la necesidad de la cooperación local, nacional e internacional en la resolución de los problemas ambientales. En línea con estos autores, el Ministerio del Medio Ambiente y el Ministerio de Educación Nacional (2002) afirman que la EA permite al individuo apropiarse de su realidad concreta y generar en la comunidad actitudes de valoración y respeto por el ambiente, a través del conocimiento reflexivo y crítico de su realidad política, ambiental y socioeconómica.

\section{El problema de la educación ambiental en}

\section{las Instituciones Educativas-IE}

Sánchez (2011) considera que uno de los problemas fundamentales de la educación ambiental ha sido la prevalencia de una formulación desarticulada de los proyectos ambientales en la educación. Y en un sentido similar, Vasco (1982) y Torres (1996) atribuyeron la inadecuada articulación a la falta de conexión entre asignaturas, es decir entre los diferentes saberes y métodos que pueden atravesar el plan de estudio. Ello implica la necesidad de un cambio en los modelos estratégicos de enseñanza actuales. 
Por otra parte, algunos procesos educativos escolares asumen la EA como un tipo de enseñanza que se concretiza, por ejemplo, mediante el Proyecto Ambiental Escolar, el cual frecuentemente es direccionado hacia el activismo ecológico, en vez de generar las ideas y los espacios para construir la educación ambiental en el marco de una formación holística para el desarrollo de los niños (Cano, 2012).

\section{La educación ambiental en las institu- ciones educativas de modalidad agropecuaria}

Mediante la Ley 99 de 1993, se creó el Ministerio del Medio Ambiente, que ordena el sector público encargado de la gestión y conservación del medio ambiente y los recursos naturales renovables, y además organiza el sistema nacional ambiental. Posteriormente, por medio de la Ley 115 de 1994, reglamentada por los Decretos 1743 y 1860 de 1994, se institucionalizó el proyecto de educación ambiental para todos los niveles de educación formal, estableciendo la responsabilidad de la comunidad educativa en el diseño y el desarrollo de los Proyectos Ambientales Educativos-PRAE, como una de las herramientas para la implementación de la educación ambiental.

De esta manera, de acuerdo con el Ministerio de Educación y el Ministerio de Medio Ambiente (2002),

La inclusión de la EA en el currículo no se hace ni a través de una materia más, ni a través de una cátedra, ni a través de una disciplina o de acciones aisladas, la EA en la reforma educativa, está concebida desde la visión sistémica del ambiente, desde la investigación pedagógica y didáctica para el tratamiento de problemas de diagnóstico ambiental particular y desde la idea de formación de dinamizadores ambientales, en el marco de procesos de cualificación conceptuales, metodológicos y estratégicos. (p.10)

Así las cosas, la estrategia de implementación de la educación ambiental en las diferentes IE es de su total autonomía, pues, son ellas las responsables de diseñar y aplicar sus propias estrategias metodológicas concernientes a la educación ambiental, así como de suplir las debilidades encontradas en los modelos tradicionales, de modo que al implementar estas nuevas estrategias se avance en la formación de una propuesta más acorde con el contexto nacional.

\section{Algunas posturas como salida al problema}

Para Pedroza y Argüello (2002), esta problemática prevalece por un ascetismo entre las áreas del saber, lo que no permite avanzar en el desarrollo de la EA. Bondarenko (2009) subraya la necesidad de dedicar esfuerzos a la formulación de teorías transdisciplinarias coherentes y lógicas, de modo que se produzcan saberes más holísticos, sinérgicos e integrales. A su juicio, la educación ambiental requiere de un modelo educativo transdisciplinario, entendiendo por tal una integración entre el conocimiento, la construcción participativa y las autoridades reguladoras (Carrizo, s.f.). 
Arqueros y Gallardo (2014) sugieren un asunto práctico para que la articulación de saberes se dé en forma fácil y didáctica; para ellos, las huertas constituyen una oportunidad para articular las diferentes disciplinas, no limitándose a contenidos de las Ciencias Naturales, Sociales o del cálculo matemático, sino también para desarrollar valores como el compromiso.

El reto de las IE en términos de educación ambiental siempre ha consistido en involucrar lo ambiental desde las diferentes asignaturas, siendo este un ejercicio transversal y transdisciplinar. Lo anterior implica un esfuerzo grande para no caer en el simple activismo ecológico o la realización de esquemas prácticos que se queden en el oficio mecánico, sin el sentido emocional y de valores que lo ambiental debe implicar.

Acorde con todo lo expresado hasta aquí, el objetivo del estudio que conllevó al presente escrito es caracterizar y analizar las percepciones de la educación ambiental de los docentes y estudiantes de la Institución Educativa Agrotécnico Mixto del municipio de Belén de los Andaquíes, departamento de Caquetá (Colombia). Se trata de un colegio de la modalidad agropecuaria, que ha venido incorporando en su trabajo elementos de la educación ambiental desde 1982, a través de huertas escolares desarrolladas en su granja San Francisco de Asís. A la fecha, se desconocen los efectos y alcances que ha tenido la EA en la institución y la forma en que los estudiantes y profesores leen esta realidad, por lo que a partir de nuestro estudio se ha elaborado una propuesta en la que se sugieran los ajustes necesarios para que la institución avance de mejor manera en el proceso integral que demanda la educación ambiental.

El escrito está dividido en tres apartados: 1. Imaginarios de los estudiantes y docentes; 2. Contraste de las percepciones entre estudiantes y docentes; y 3. Diseño de una propuesta pedagógica a partir de los resultados obtenidos, que retome las fortalezas del sistema adoptado según la perspectiva de los participantes e incluya aspectos que la complementen.

\section{Método}

\section{Localización}

La investigación se realizó en la Institución EducativaAgrotécnico Mixto, ubicada en el casco urbano del municipio de Belén de los Andaquíes, departamento de Caquetá (Colombia). El municipio se encuentra localizado al sur del país a 1²4'59" latitud Norte y 75'52'21" longitud Oeste, con una altura promedio de 312 metros sobre el nivel del mar, aproximadamente a 43 kilómetros de la ciudad de Florencia, capital del departamento. Su cabecera está localizada en el piedemonte de la cordillera oriental a la margen derecha del río Pescado, tiene un área aproximada de 1111 kilómetros cuadrados, que hace parte de la cuenca Amazónica (Alcaldía de Belén de los Andaquíes, 2012).

\section{Población objeto del estudio}

El estudio involucró dos tipos de población: por un lado, los estudiantes de la IE Agrotécnico 
Mixto, quienes, además de ser jóvenes campesinos de las zonas rurales aledañas a la cabecera municipal y llevar un proceso de formación en los núcleos básicos exigidos por la educación media, han recibido instrucciones en las asignaturas propias de la formación en las áreas pecuarias, agrícolas, agroecológicas y ambientales. De esta manera y como criterio de selección de los informantes clave, se tuvo en cuenta que fueran estudiantes de los grados 10 y 11, pues, por estar a punto de culminar su proceso de formación, se consideran con mayor madurez y conocimiento. De igual forma, se consultó a los docentes de la institución, responsables de las asignaturas relacionadas con las áreas agropecuarias propias de la especialidad institucional.

\section{Enfoque metodológico}

$\mathrm{La}$ investigación se fundamenta en el paradigma de investigación crítico social, puesto que se desarrolla desde la praxis de los actores y se analizan sus percepciones mediante el empoderamiento social, para realizar aportes reflexivos que pueden cambiar la realidad de los agentes involucrados (Alvarado \& García, 2008). Siguiendo a Byrman (1988), además, nuestro trabajo es de corte cualitativo, toda vez que la relación entre el investigador y el sujeto depende de los cánones de comprensión, en el entendimiento de que la realidad es socialmente construida por los miembros de la sociedad y, por otra parte, es de carácter inductiva porque busca comprender los ejes que orientan el compromiso. El tipo de investigación es diagnóstica-comparativa, pues, analiza el conocimiento estudiantil y de los docentes sobre la educación ambiental y el manejo de las huertas de la granja; por la naturaleza de los datos, se trata, como ya se ha dicho, de una investigación de corte cualitativo, pues, las variables no son medibles y el estudio está orientado al abordaje de la realidad social institucional.

La presente investigación se fundamenta, por último, en el método de investigación hipotético-deductivo, reconociendo a este como el conjunto de instrucciones que, al ser guiadas cuidadosamente, permiten ensamblar la realidad que se estudia (Bonilla \& Rodríguez, 1997). Se desarrollaron tres fases en la investigación: fase 1. Gabinete; fase 2. Trabajo de campoproducción de los datos y fase 3 . Triangulación.

\section{Fase 1: Gabinete: Revisión de información} secundaria

En la recolección de información se acudió a la técnica de revisión de archivo, consultándose documentales impresos y digitales acerca de la EA, disponibles tanto en la web como en sus documentos internos. Las variables de interés en esta búsqueda fueron: huertas escolares como estrategia pedagógica, transdisciplinariedad y currículo en EA. La información fue catalogada de acuerdo con el nivel de similitud o contrariedad.

Fase 2: Trabajo de campo, producción de los datos

Caracterización del imaginario de estudiantes y profesores

Para conocer la percepción de los estudiantes 
y profesores acerca del ambiente, la educación ambiental en la IE y lo que debería llevar una estrategia de aprendizaje en una IE con modalidad agropecuaria, se utilizó como instrumento de recolección de información una entrevista semiestructurada con cada uno de los actores, considerando temas relativos a la educación ambiental, como: la importancia de la formación integral del estudiante, el concepto de ambiente y de educación ambiental, las estrategias para mejorar la educación ambiental, las huertas como herramienta de aprendizaje y conocimientos técnicos, asumiendo la entrevista como un instrumento que permite recopilar las experiencias vividas por los maestros dentro y fuera de la escuela y sus trayectorias profesionales (Albor \& Villamil, 2012).

Dichas entrevistas se grabaron con previa autorización de los interesados, luego fueron transcritas y, con la información obtenida se procedió a elaborar un texto plano, en el que se marcaron las frases importantes y se les dio un código estratégico que permitía definirlas $\mathrm{y}$ posteriormente ordenarlas en familias en categorías de análisis. Lo anterior con la ayuda del procesador de datos ATLAS TI versión 7.5.4.

Fase 3: Triangulación para la elaboración de la propuesta "Caminando a una estrategia conjunta de aprendizaje ambiental"

A partir del análisis de las percepciones de la comunidad educativa sobre la EA, en esta última instancia se diseñó una propuesta, la cual busca enmarcar las debilidades, fortalezas y poten- cialidades que evidenciaron los actores con el propósito de mejorar el diseño metodológico de la educación ambiental en la IE.

\section{Resultados}

\section{El concepto de ambiente}

Los estudiantes reconocen el ambiente como un escenario donde se dan las "interacciones" ecológicas entre los seres vivos y no vivos; también como el espacio, conjunto o lugar que permite acceder a "materias primas" para satisfacer las necesidades humanas. El discurso estudiantil al respecto refleja un imaginario bastante moderno de la naturaleza, donde se diferencia lo natural de lo humano en el ambiente, y el ser humano actúa e interactúa con lo natural, ya sea como el beneficiario, el "protector" o "abusador" ilegal de la "biodiversidad" (Figura 1 , resaltado con cursiva y viñetas).

Siguiendo a Palsson (citado en Ulloa, 2001), la relación moderna naturaleza/cultura consiste en "conocer" la naturaleza a través de la explotación y la protección; y Ulloa precisa que la explotación es orientalista y la protección, paternalista. La explotación como la implicación de la apertura de fronteras y la expansión del control humano mediante la domesticación de lo natural, y la protección, referida a la responsabilidad humana por el cuidado de otras especies. En ambas situaciones, los humanos son los amos, dueños de una naturaleza que es objeto de domesticación y protección, por ser considerada como algo inferior y asociada a la animalidad (Ulloa, 2001). 
El concepto de ambiente expresado por los estudiantes coincide entonces con las definiciones de González (1999), quien lo entiende como todos los factores (vivientes y no vivientes) que nos rodean y que afectan directamente a los organismos (como nosotros). También coincide con la definición propuesta en Estocolmo en 1972, en la que se manifiesta que el ambiente es “el conjunto de componentes físicos, químicos, biológicos y sociales capaces de causar efectos directos e indirectos, en un plazo corto o largo, sobre los seres vivos y las actividades humanas" (Organización de las Naciones Unidas, citado por Sabalain, 2009, p.12).

Los docentes entrevistados entienden el mente, una holística (Martínez, 2010).

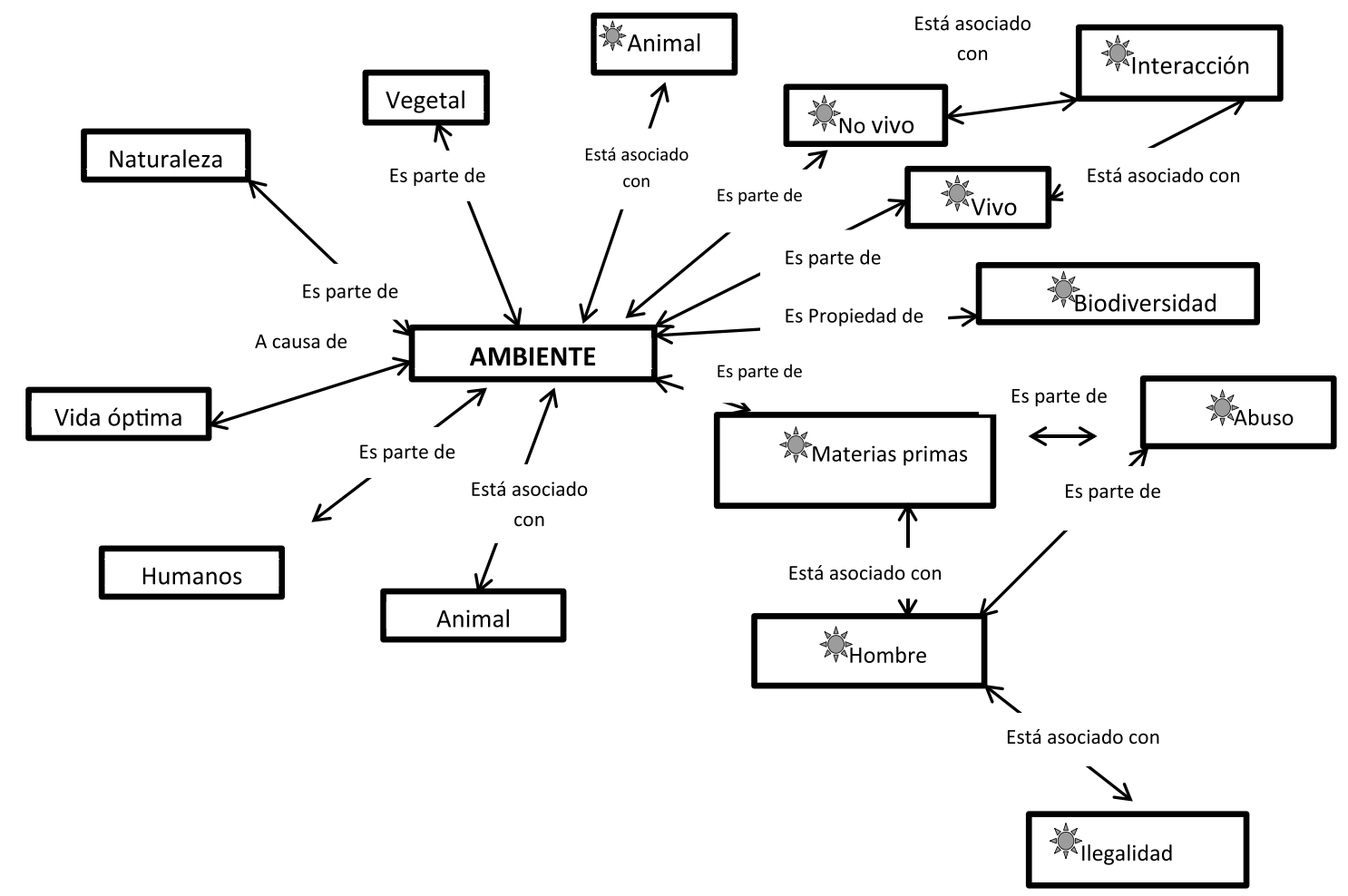

Figura 1. Imaginario estudiantil acerca del ambiente Fuente: Elaboración propia 
La educación ambiental en las IE de modalidad agropecuaria

Al consultar a los estudiantes y docentes acerca de la relación de la modalidad de su colegio con el manejo de los problemas ambientales, como ellos los habían descrito (deforestación, abuso, ilegalidad, contaminación, basuras e incendios), estos dejaron ver en su relato categorías de análisis asociadas a la importancia de la educación ambiental. En concreto, para ellos, esta debería orientarse tanto al "aprendizaje" como hacia la capacidad de "enseñar". De igual forma, el proceso operativo de la educación debería buscar los mecanismos para no limitarse al "cuidado" del "ambiente", sino también el "ser humano"; para ello no solo se requiere el dominio de conceptos técnicos, sino de rescatar los valores, es decir, el ambiente asociado con la garantía de tener "vida" en "familia" y la familia como parte integral de este. La educación aparece entonces como una estrategia de cambio, lo cual coincide con lo planteado por González, Gómez y Sánchez (2016). No obstante, el cambio debe ser abordado desde la "transversalidad" curricular, y la "practicidad", con acciones concretas como reforestación, concientización, cuidado y el pensar no solo en el ser individual, sino en el nosotros (Figura 2).

La educación ambiental se concibe, así, como un deseo por el cuidado del medio ambiente, la mitigación del impacto negativo en este, asumiéndose el conocimiento en relación con el uso sostenible del ambiente. En este sentido, Herre-

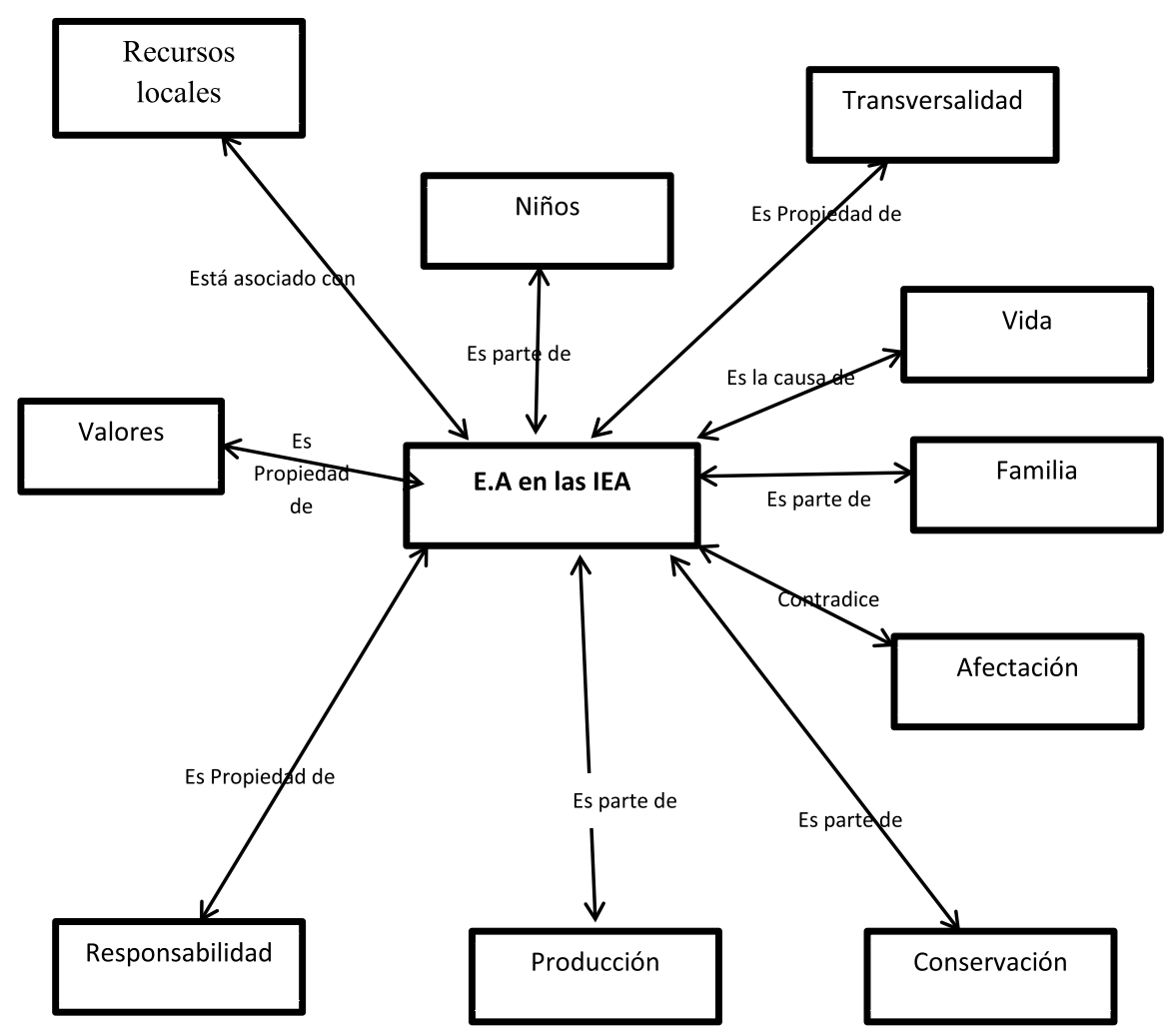

Figura 2. Percepción estudiantil acerca de la Educación Ambiental en la IE Fuente: Elaboración propia 
ría y López (1996) consideran que la fragmentación del conocimiento y la enseñanza dificulta la comprensión de los fenómenos, porque el aislamiento de múltiples facetas limita su funcionamiento global, lo cual hace que aun cuando la información esté en manos del estudiante, este no sepa qué hacer con ella y no logre conectar los conceptos con la realidad.

Las categorías identificadas evidenciaron la EA como una estrategia instrumental para mejorar las condiciones del ambiente y asegurar la vida de una sociedad. En este sentido, las percepciones acerca del deber ser de la educación ambiental se encaminaron sobre todo hacia la conservación y protección, un asunto meramente intervencionista, olvidando los principales ejes de la educación ambiental propuestos por la UNESCO: el pensamiento crítico y el enfoque de solución de problemas, lo cual debe iniciarse de manera temprana en la escuela (UNESCO, 1997). Así las cosas, sus discursos parecieran estar desligados de la producción, pues en sus expresiones no se logró establecer una conexión entre los elementos citados y el fin de una producción sostenible o agroecológica, que al final es el objetivo de la modalidad de la IE.

La educación ambiental se percibe en este contexto como una herramienta instrumental para solucionar los problemas ambientales y no como una modalidad de vida. Al respecto, Rengifo et al. (2012) plantean que ante la situación actual del ambiente, urge una toma de conciencia que genere nuevos estilos de vida, de manera que haya interacciones armónicas entre los seres humanos y entre estos con otras formas de vida.
La EA debe contemplar un contexto amplio en el que se forjen valores, y se desarrollen competencias y conocimientos que propicien soluciones para los problemas ambientales (Camacho \& Marín, 2011).

Por otra parte, los docentes recalcan la transversalidad de la educación ambiental en las diferentes materias con una transdisciplinariedad, para así formar y fortalecer al estudiante de manera completa; mencionan que esto es lo que hacen en la institución. En este sentido, Smith (1997) afirma que la educación ambiental no es un campo de estudio, como la biología, química, ecología o la física, sino que es un proceso; por tanto, se pueden enseñar conceptos de educación ambiental, pero no educación ambiental. Sin embargo, a pesar de lo afirmado por los docentes, no se está haciendo una transdisciplinariedad en las asignaturas, ya que el tema se le atribuye principalmente a la asignatura de las Ciencias Naturales. Como plantean Pedroza y Argüello (2002), la educación ambiental aborda de manera general las disciplinas en las que se enseña la cuestión ambiental, mostrando como, con la transdisciplinariedad, se intenta superar las barreras de las disciplinas.

Los docentes piensan que su enseñanza es tan solo una parte de la formación del estudiante, y que dicha formación debe trascender desde la familia. Coinciden en ello con Álvarez y Vega (2002), para quienes la inserción de temáticas ambientales en el sistema educativo, se facilita cuando se vincula con la formación inicial familiar, aun cuando, como indican Shymansky y Kyle (1992), el profesor sigue siendo un agente clave en el proceso práctico de las innovaciones curriculares en cualquier sociedad. 


\section{Las estrategias de educación ambiental}

La huerta escolar, como estrategia de enseñanza de educación ambiental y no como simple herramienta de aprendizaje en producción, propicia la construcción de conocimiento a través de la relación sujeto-objeto de estudio. De esta manera se desarrollan experiencias significativas y transformadoras, encontrándose el maestro ante el desafío de compartir la jornada de trabajo con estudiantes interesados por el aprendizaje (Vera, 2015).

Los docentes reconocen la enseñanza como la mejor estrategia para interiorizar la educación ambiental, y ven a los padres como actores estratégicos para su inculcación; también resaltan que el interés de los estudiantes es esencial para conseguir su objetivo: "lograr que el alumno entienda que el medio ambiente se debe conservar de una manera y sin afectar su medio social". Precisamente, la legislación nacional estipula que, a través del gobierno escolar, las instituciones educativas deben implementar un modelo democrático y participativo, que fomente la participación activa, organizada $y$ responsable de padres, docentes, estudiantes, exalumnos y sector productivo, con el fin de garantizar un desarrollo adecuado de alumnos $\mathrm{y}$ alumnas (MEN, 2007).

Los docentes reconocen la necesidad de una interiorización de saberes de educación ambiental y enfocan unas alternativas para los estudiantes. Para dichos docentes la conciencia no solo implica ser consciente, sino la representación y asunción de valores respecto al ambiente, resaltando que es aquí cuando entra la educación ambiental de manera holística. En la EA es muy importante la reflexión y el desarrollo de la conciencia, debido a las acciones de depredación que se presentan en la sociedad (hombre consigo mismo, con los demás y con el medio que los rodea), donde prevalece la indiferencia y el mal uso de los recursos naturales (Maciel, 2009).

Según Cardona y Jiménez (2009), el problema ambiental tiene su origen en la escasa formación de los profesores para abordar la EA como área transversal, por esto presentan dificultades para incluir cuestiones ambientales en los contenidos de las asignaturas y reconocen su responsabilidad. En este sentido, los profesores perciben la huerta como herramienta metodológica para la educación ambiental, pero solo enseñan la conservación al ilustrar en particular la producción orgánica; sin embargo, esta metodología solo evita el consumismo y no ayuda en la formación integral del estudiante. De acuerdo con González et al. (2014), lo ambiental ha tenido muchos puntos de partida y de debate, sin embargo, la mayoría de estas discusiones se han quedado en una concepción ecologista dirigida a la protección y conservación de los ecosistemas y no tienen en cuenta los componentes sociales, físicos y culturales. De ahí que los huertos deban ser utilizados en todas sus potencialidades, porque de este modo es más factible que ejerzan cambios significativos en los valores y las actitudes de las personas hacia el medio ambiente (García, 2009). Lo anterior no significa que una estrategia de educación ambiental funcional en una IE de modalidad agropecuaria deje de lado el interés económico, pues solo a partir de la evaluación de la sustentabilidad y 


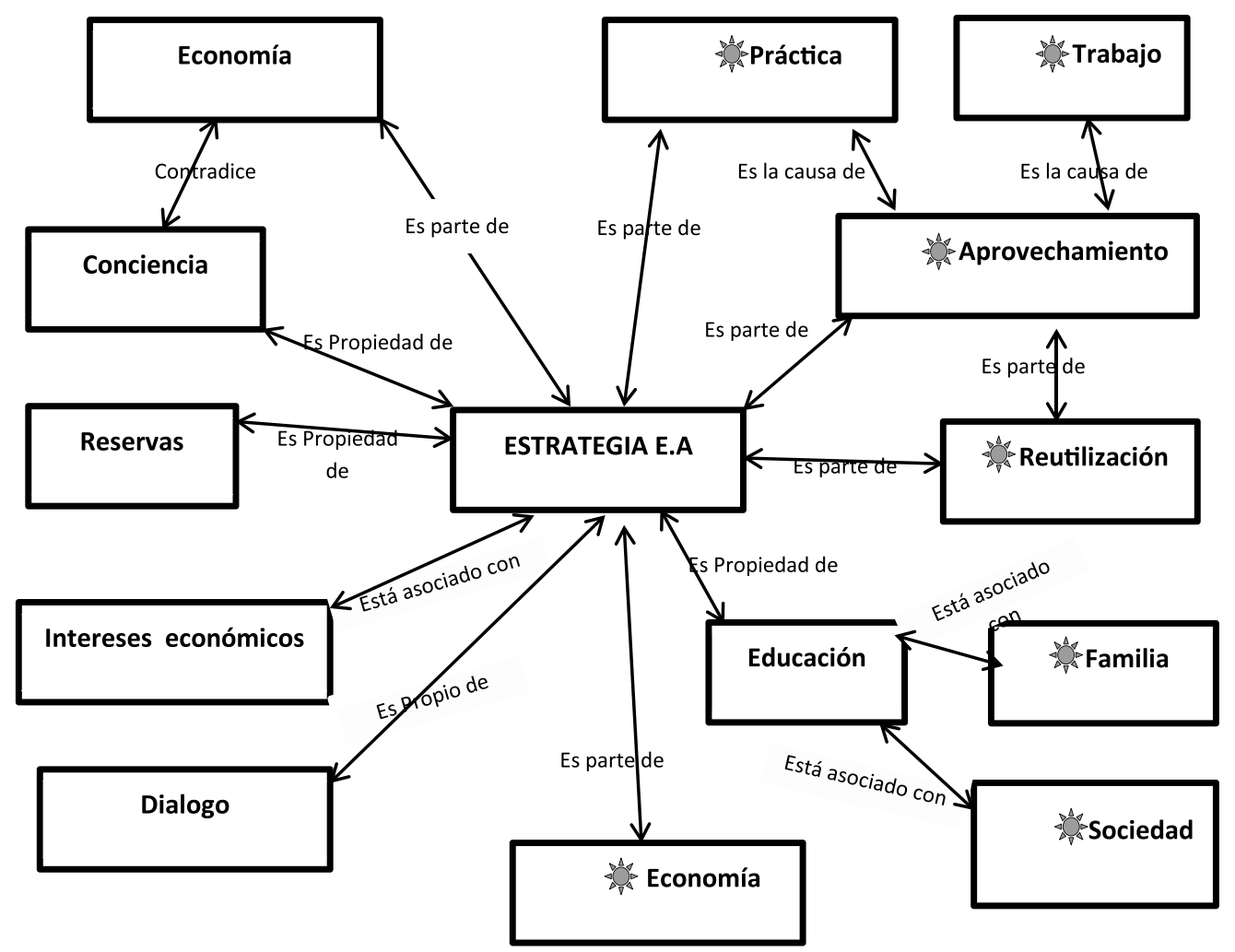

Figura 3. Percepción docentes y estudiantes de la estrategia de educación ambiental Fuente: elaboración propia

viabilidad del modelo, se podrá exteriorizar el modelo mismo hacia fuera (Figura 3. Categorías sin viñetas. Percepción docente).

Para los estudiantes de la IE, como ya se ha escrito, la estrategia debe ser pensada para una sociedad y no solo para los estudiantes, involucrar más a las familias y desarrollar procesos de aprovechamiento, lo cual implica más práctica y trabajo (Figura 3. Viñetas. Percepción estudiantil).

\section{Discusión}

La educación ambiental en las huertas desde el docente y el estudiante

La percepción de los estudiantes acerca de la educación ambiental en las huertas está sesgada hacia lo productivo, en donde para llevar a cabo este proceso se hace un aprovechamiento de los recursos de la granja con procedimientos de reutilización y compostaje. Ello supone una gran discrepancia con los imaginarios de los docentes, ya que estos las perciben como una herramienta pedagógica que enmarca una totalidad de conocimientos y debe ayudar a la formación del estudiante mediante procesos orientados con pertenencia, cuidado y un mejor direccionamiento hacia el medio ambiente.

Esto enmarca un problema, pues, la información que los docentes quieren inculcar en los estudiantes no se está alcanzando de la manera adecuada. En este sentido, según Trahtemberg 
(citado por Paniagua, 2004), "se requieren cambios paradigmáticos para girar del tradicional estilo pedagógico centrado en el profesor, la enseñanza, la información, hacia un nuevo enfoque centrado en el estudiante, que sea capaz de pensar en asuntos que vayan más allá de las disciplinas o de sus áreas de especialización" (p.5). El desempeño educativo debe concebirse desde una visón renovada e integral y entenderse como "el proceso de movilización de sus capacidades profesionales, su disposición personal y su responsabilidad social para articular relaciones significativas entre los componentes que impactan la formación de los alumnos" (Robalino, 2005, p.3).

De todos modos, los profesores reconocen el reciclaje como una estrategia para evitar el deterioro del medio ambiente. Además, optar por las huertas como herramienta pedagógica-práctica comprende un potencial académico muy alto, lo que ha hecho, por ejemplo, que los estudiantes adquieran experiencias de compostaje y, por ende, su entendimiento claro. En esta medida, el huerto escolar se puede potencializar de una manera eficiente cuando el fin último es la generación de experiencias reflexivas y no solo una vivencia práctica, ya que supone un cambio de estrategias docentes para producir una EA adecuada y se considere como una innovación (Ripoll, Ballster \& Rosa, 1998).

\section{La educación ambiental desde el docente y} el estudiante en las asignaturas

Los estudiantes reconocen el impacto del ser humano en la naturaleza. Por esta razón, se sien- ten insatisfechos y perciben la necesidad de una orientación amplia en el contexto de EA. Advierten, en efecto, que la educación ambiental divaga en las diferentes materias como un complemento, pero solo se la atribuyen a las áreas de pecuarias, administración y biología, por ser las que presentan más afinidad con esta. Esto contrasta con lo mencionado por los docentes, que se refieren a una transdisciplinariedad de la EA en las diferentes materias, aunque reconocen la necesidad de una reestructuración pedagógica. El Ministerio de Educación Nacional (2002) afirma al respecto que la explicación de un fenómeno se soporta con argumentos de varias disciplinas del saber, pero que la explicación global solo se da cuando se integran dichos argumentos; de lo contrario, se creará una ambigüedad que puede dificultar el entendimiento del fenómeno.

\section{Actores en la formación integral del estu-}

\section{diante}

Los estudiantes consideran que el aprendizaje de educación ambiental en su formación académica y personal está relacionado con los métodos que utilice la institución en las diferentes asignaturas y herramientas, como lo son las huertas escolares. No obstante, piensan que esto depende de los docentes, ya que son quienes direccionan el modelo de aprendizaje que ellos van a adquirir. Por su parte, los educadores resaltan que es difícil llevar este proceso solos, debido a que se requiere un interés del mismo estudiante para asimilar la pedagogía impartida.

Asimismo, admiten que la institución aporta parte del aprendizaje que adquieren de la 
educación ambiental, actuando esta como un complemento indispensable para su formación, pero también asumen que el hogar constituye un complemento básico y esencial. Según Barraza (1998), la primera institución de educación no formal es el hogar, por esta razón, si los padres están comprometidos con el respeto y cuidado del ambiente y la naturaleza en todas sus formas, y llevan a la práctica acciones concretas en casa, como la separación de la basura, es probable que sus hijos también lo hagan. Molina (2003) considera al respecto que los estudiantes que no alcanzan los objetivos planteados pueden atribuirlo a una falta de motivación debida a una divergencia entre la cultura escolar y la cultura familiar o social.

Reconocimiento de los problemas ambientales

Estudiantes y docentes tienen una visión similar sobre el deterioro del medio ambiente. Sin embargo, ambos poseen perspectivas diferentes con relación a los principales actores de este problema: los docentes atribuyen y dimensionan esta problemática a un nivel global, apuntando como principales actores a la tecnología y a las grandes industrias, entendiendo estas como la globalización. En contraste con ello, en los estudiantes predomina una perspectiva local y dimensionaron el deterioro ambiental con la deforestación del municipio, causada por las mismas personas de la región movidas por la ambición y la necesidad.

El reconocimiento de la problemática por parte de los estudiantes, a partir del orden local del deterioro del medio ambiente es bueno, porque según Giordan y Souchon (1992), cuando se generaliza algo influido por los medios, estos desinforman "banalizando el tema o insuflando la ilusión de un saber, porque el público cuando repite palabras como polución, efecto de cierre, agujero de ozono, tiene la ilusión de saber, pero infortunadamente este saber no es operativo" (p.17). Por lo tanto, reconocer algo tras la observación propia es bueno, porque permitirá una concientización más efectiva y rápida por parte de los estudiantes.

\section{Hacia una estrategia conjunta de aprendi-} zaje ambiental

Los hallazgos obtenidos permitieron reconocer las debilidades y las fortalezas del sistema educativo de la institución estudiada, y a partir de todo ello desarrollamos una propuesta cuyo propósito consiste en aportar al mejoramiento del sistema metodológico de la educación ambiental en la IE. Esta propuesta se ha de concretar en fases.

Fase 1. Unificación de la apuesta institucional

En esta fase se busca que los diferentes actores definan e interioricen de manera colectiva la meta de la institución en términos de su modalidad, es decir, que precisen cuál será el sello de la IE Agrotécnico Mixto. Una vez quede clara la apuesta institucional, se seguirá con la socialización de la línea base y la sensibilización ambiental. 
Fase 2. Definición de líneas pedagógicas vinculantes con la agroecología

Esta parte implica la participación de docentes y directivos de la institución. Se busca que cada uno de ellos socialice los ejes temáticos que abordan en cada grado y en las diferentes asignaturas. A partir de ello, se identificará qué parte y de qué manera de los asuntos propios de la educación ambiental se podría trabajar o se está trabajando en la escuela, interiorizando en cada asignatura la importancia de la producción agroecológica y la visión de sistemas.

Las materias o asignaturas serán agrupadas por afinidad, definiendo ejes agroecológicos para el trabajo educativo, y cada eje o línea pedagógica tendrá un plan de trabajo, con metas y responsables.

Fase 3. Incorporación de la temática en los microcurrículos

Con la apuesta institucional clara y los ejes agroecológicos definidos en las respectivas asignaturas, se procederá a incorporar en los microcurrículos las temáticas requeridas en cada uno de los grados, las cuales guardarán una secuencia desde el sexto hasta el once grado.

\section{Fase 4. Diseño de prácticas integrales}

Por grado, se diseñarán prácticas de campo, que permitan abordar de manera integral varios escenarios ambientales y de la producción agroecológica.

Por ejemplo, en los resultados obtenidos se apreció que la huerta funciona como el principal recurso de la institución para la enseñanza; por ende, esta se ha de reforzar, para que la propuesta logre ajustarse al sistema de enseñanza de la IE. El ajuste comprende la transición de una huerta orgánica a una huerta agroecológica, encerrando los diferentes matices de la educación ambiental con enfoque en los diferentes campos sociales, culturales, ambientales y éticos, y desarrollando las actitudes y aptitudes de los estudiantes.

La propuesta busca aprovechar la enseñanza tradicional, en la cual se imparten conocimientos para la elaboración y preparación de una huerta, pero de modo que esta trascienda a un conocimiento dinámico, que enmarque las diferentes competencias y vincule la transdisciplinariedad.

\section{Resultados esperados}

La implementación de esta propuesta comprende una metodología activa y constructiva, que busca generar aspectos como:

a. Desarrollo de habilidades. Por medio de la interacción constante con sus compañeros y la relación con el medio ambiente, los estudiantes aprenderán a desarrollar una empatía y una comunicación asertiva, a fin de fortalecer las relaciones interpersonales, así como a manejar las emociones y sentimientos, los problemas y conflictos, $\mathrm{y}$ a incentivar un pensamiento creativo y crítico.

b. Fortalecimiento de la transdisciplinariedad. El estudiante entenderá la huerta agroecológica como un campo amplio de conocimiento, donde se articulen las diferentes materias y se trabaje en pro del mismo objetivo.

c. Organización y creatividad. En un portafolio 
hecho con materiales reciclados, se archivarán todas las actividades y experiencias adquiridas, con el fin de realizar su sistematización, que ayude en el proceso reflexivo de la práctica pedagógica.

d. Fortalecimiento del autocuidado. La educación ambiental irá de la mano con la personalidad humana (me cuido, cuido mi medio).

e. Articulación. Se realizarán talleres entre padres y estudiantes, que incluyan actividades en casa y en el colegio a fin de forjar puentes entre estos actores y generar una experiencia significativa. Del mismo modo, se trabajará en la concepción de una producción agroecológica y se socializarán los aprendizajes por medio de jornadas de limpieza en las zonas aledañas al colegio, integrando la comunidad.

f. Emprendimiento. Al entender las diferentes potencialidades de la huerta agroecológica, el estudiante despertará entusiasmo por la investigación.

\section{Conclusiones}

La estrategia metodológica para la enseñanza de la educación ambiental refiere un modelo pedagógico tradicional, el cual es ajeno a la articulación entre las diferentes áreas del saber. Lo anterior conlleva a que la educación ambiental sea vista como un agente exclusivo de las áreas relacionadas con las Ciencias Naturales, dejando de lado la integración de los demás espacios académicos y limitando la transdisciplinariedad propia de un proceso de educación ambiental integral, es decir, en el que todas las áreas del saber permitan la construcción colectiva del tema.
En los imaginarios acerca de la educación ambiental, existen diferencias por parte de los docentes y estudiantes, puesto que estos últimos perciben la EA como una manera de reutilizar a partir de conceptos ecológicos, y piensan que ello se puede lograr mediante el diálogo y la concientización; en tanto que los docentes la ven como un modelo de enseñanza en el cual se educa a los estudiantes para que cuiden el ambiente, trabajando de una manera transversal entre las diferentes asignaturas.

En consecuencia, los imaginarios ambientales y de educación ambiental tanto en docentes como en estudiantes son reconocidos especialmente en acciones prácticas que separan la conservación de la producción. Aunque la agroecología se menciona en los discursos, no se percibe una interiorización por parte de los entrevistados, pues las acciones de aprendizaje están desconectadas de los principios de producción y conservación.

La huerta escolar es concebida como una herramienta de aprendizaje y conocimiento técnico, mas no como una estrategia pedagógica que permita la integración de las diferentes áreas ni la vinculación de padres de familia, docentes y estudiantes. En adelante, esta deberá ser vista como un mecanismo dinamizador del conocimiento, el desarrollo de habilidades y la generación de conciencias sobre la producción agroecológica.

Si se desea llevar a cabo un verdadero proceso de enseñanza y aprendizaje de la educación ambiental, conforme a las exigencias reglamen- 
tarias y misionales de las instituciones de educación media con énfasis agropecuario, esta deberá desarrollarse de manera transversal en todos los espacios de formación, privilegiando la articulación de lo académico y la búsqueda, implementación y apropiación de modelos productivos más sostenibles e incluyentes.

\section{Referencias}

Albor, C, \& Villamil, L. (2012). Una mirada a las prácticas pedagógicas de los docentes en Colegios Públicos de Barranquilla y Girón (Departamento de Santander) para intervenir los problemas de violencia escolar. Revista Educación y Humanismo, 14 (23), 196-215.

Alcaldía Municipal de Belén de los Andaquíes. (2012). Plan Municipal de Desarrollo 2012-2015: Organización social y Comunitaria por Belén. Municipio de Belén de los Andaquíes. Caquetá (Colombia).

Alvarado, L. García. M. (2008). Características más relevantes del paradigma sociocritico: su aplicación en investigaciones de educación ambiental y de enseñanza de las ciencias realizadas en el Doctorado de Educación del Instituto Pedagógico de Carcas. Revista Universitaria de Investigación, 9 (2). 187-202

Álvarez, S., Vega, M. (2002). Formación inicial del profesorado en educación ambiental. ¿Para qué, cómo hacerla?: Presentación de una estrategia metodológica. España: Centro Superior de Educación. Universidad de la Laguna.

Arqueros, M., Gallardo, N. (2014). La huerta agroecológica como proceso de ense- ñanza-aprendizaje. Disponible en: http:// cienciahoy.org.ar/2014/08/la-huerta-agroecologica-como-proceso-de-ensenanzaaprendizaje/, Consultado en 24.08.2016.

Barba, T. Sanzetencea, J. (2002). La educación ambiental y la comunidad educativa. En: Textos escogidos en educación ambiental. Tomo I. Coord: Sauvé, L., Orellana, I., Sato Michele. Québec (Canadá): Les Publications ERE-UQAM..

Barraza, L. (1998). Conservación y medio ambiente para niños menores de 5 años. En: Revista sobre conservación y biodiversidad, vol. 7. México.

Byrman, A. (1988). Quanty and quality in social research. London, Boston. Unwim Hyman Publications.

Bondarenko, N. (2009). El concepto de teoría: de las teorías intradisciplinarias. En: Revista de Teoría y Didáctica de las Ciencias Sociales, $n^{\circ} 15$. Mérida (Venezuela).

Bonilla C, E. Rodríguez S, P. (1997). La investigación en ciencias sociales, más allá del dilema de los métodos. Bogotá: Grupo Editorial Norma. Ediciones Uniandes.,

Camacho, R., Marín, X. (2011). Tendencias de enseñanza de educación ambiental desde las concepciones que tienen los maestros en sus prácticas escolares. Requisito para optar el título de Licenciatura en Educación Básica con énfasis en Ciencias Naturales y Educación Ambiental. Instituto de Educación y Pedagogía. Cali (Colombia)

Cano S, L.C. (2012). La educación ambiental en la básica primaria: perspectivas desde la teoría ecológica de Urie Bronfrenbrenner. Tesis de maestría en medio ambiente 
y desarrollo. Universidad Nacional de Colombia-Sede Manizales. Manizales (Colombia).

Cardona, J.D., Jiménez, R. (2009). Las concepciones iniciales del profesorado de educación básica y media sobre la educación ambiental. En: Tecné, Episteme y Didaxis: TED No. Extraordinario. $4^{\circ}$ Congreso Internacional sobre Formación de Profesores de Ciencias.

Carrizo, L. (sf). Pensamiento complejo y trasndisciplinariedad. Disponible en: http:// www.upch.edu.pe/rector/durs/images/ Biblio/MarcoConceptual/PensamientoComplejoTransdisiplinario/pensamientocomplejoytransdisciplinariedad.pdf. Fecha de consulta: agosto 27 de 2016.

Congreso de la Republica de Colombia. (1993). Ley 99 "Por la cual se crea el Ministerio del Medio Ambiente, se reordena el Sector Público encargado de la gestión y conservación del medio ambiente y los recursos naturales renovables, se organiza el Sistema Nacional Ambiental, SINA, y se dictan otras disposiciones". Diario Oficial 41146 de diciembre 22 de 1993. Santafé de Bogotá (DC), Colombia.

Congreso de la Republica de Colombia. (1994). Ley 115 "Por la cual se expide la Ley General de Educación". Diario Oficial 41.214 del 8 de febrero de 1994. Santafé de Bogotá (DC), Colombia.

Descola, P., Palsson, G. 2001. Naturaleza y sociedad: perspectivas antropológicas. México: Siglo XXI.

García, M.E. (2009). El huerto escolar como herramienta pedagógica en la educación ambiental. Requisito para optar el título de Maestría en Artes en Estudios Ambientales en Educación Ambiental. Universidad Metropolitana. San Juan (Puerto Rico)

Giordan, A., Souchon, C. (1992). Une education pour l'environnement. Disponible en https://www.erudit.org/revue/cgq/1994/ v38/n103/022420ar.pdf. Recuperado el 23.08.2016.

González H, G., Gómez C, C.A., Sánchez C, V. (2016). La educación y la gestión ambiental en la industria azucarera: una experiencia comunitaria en la localidad de la Adela-Cuba. En: Revista FACCEA. 6 (2), Universidad de la Amazonia, Florencia Caquetá (Colombia).

González, E. (1999). El ambiente: mucho más que ecología. El Universal. Suplemento de Niños. Academia Nacional de educación nacional. Caracas (Venezuela).

González, V. M., Giraldo R., Estrada. A. (2014). Educación ambiental y extensión universitaria: una mirada crítica del currículo. Trabajo presentado para optar al título de Licenciado en Educación Básica con Énfasis en Ciencias Naturales y Educación Ambiental. Facultad de Educación. Universidad De Antioquia. Medellín (Colombia).

Herrería, F., López, M. (1996). Implicaciones metodológicas de la transversalidad en la educación secundaria obligatoria. En: Revista interuniversitaria de formación del profesorado, $n^{\circ} 27$. España.

Latour, B. 2007. Nunca fuimos modernos. México: Siglo XXI. 
Maciel, F.B. (2009). Educación ambiental y turismo. Una formación holística, interdisciplinaria y de futuros educadores. En: Revista Estudios y Perspectivas en Turismo, vol. 18, $n^{\circ}$ 1. Buenos Aires (Argentina).

Martínez, R. (2010). La importancia de la educación ambiental ante la problemática actual. En: Revista Electrónica Educare, vol. $14, n^{\circ}$ 1. Costa Rica.

Meseguer, J.L, Más, D., Gil, J.L., Hernandéz, J. Guilabert, P.M. (2009). Definición, principios e historia de la Educación ambiental. Guía de trabajo, curso 2009-2010. El portal de la educación ambiental.

Ministerio De Educación Nacional -MEN. (2007) ¿Cómo participar en los procesos educativos de la escuela? Primera edición. Guías n²6. Bogotá: Autor.

Ministerio de Educación Nacional y Ministerio de Medio Ambiente. (2002). Política Nacional de Educación Ambiental SINA. Bogotá: Autor.

Molina, S. (2003). Representaciones mentales del profesorado con respecto al fracaso escolar. En: Revista interuniversitaria de formación del profesorado, vol. 7, $n^{\circ} 1$. (España).

Organización de las Naciones Unidas para la Educación, la Ciencia y la Cultura -UNESCO (1997). Actividades de la Educación ambiental para las escuelas primarias. Santiago de Chile: Centro internacional de Educación para la Conservación para el Programa Internacional de Educación Ambiental- PIEA.
Paniagua, M. (2004). La formación y la actualización de los docentes: herramientas para el cambio en educación. Centro de estudios democráticos de América Latina. Costa Rica

Pedroza, R., Argüello, F. (2002). Interdisciplinariedad y Transdisciplinariedad en los Modelos de Enseñanza de la Cuestión Ambiental. En: Revista de Epistemología de Ciencias Sociales, $n^{\circ} 15$. Veracruz (México).

Perafan, A. 2013. Quinuas, redes y nuevas significaciones en contextos del "desarrollo": el caso del Rosal, Cauca. Tesis, Doctorado en Antropología, Universidad del Cauca, Popayán

Presidencia de la República de Colombia. (1994). Decreto 1743 "Por el cual se instituye el Proyecto de Educación Ambiental para todos los niveles de educación formal, se fijan criterios para la promoción de la educación ambiental no formal e informal y se establecen los mecanismos de coordinación entre el Ministerio de Educación Nacional y el Ministerio del Medio Ambiente". Diario Oficial No. 41.476 de 03 de agosto de 1994. Santafé de Bogotá (DC), Colombia.

Presidencia de la República de Colombia. (1994). Decreto 1860 "Por el cual se reglamenta parcialmente la Ley 115 de 1994, en los aspectos pedagógicos y organizativos generales". Diario Oficial No 41.473 de 5 de agosto de 1994. Santafé de Bogotá (DC), Colombia.

Presidencia de la República de Colombia. (2005). Decreto 1286 "Por el cual se 
establecen normas sobre la participación de los padres de familia en el mejoramiento de los procesos educativos de los establecimientos oficiales y privados $y$ se adoptan otras disposiciones". Diario Oficial 45.893 de abril 28 de 2005. Santafé de Bogotá (DC), Colombia

Rengifo, B., Quitiaquez, L., Mora, Fr. (2012). La educación ambiental una estrategia pedagógica que contribuye a la solución de la problemática ambiental en Colombia. XII Coloquio de Geocrítica. Bogotá (Colombia)

Ripoll, V., Ballster C., Rosa, M. (1998). Proyecto de educación ambiental: El huerto escolar ecológico. Modelo de proyecto técnico didáctico de un huerto escolar. Valencia (España).

Robalino, M. (2005). ¿Actor o protagonista? Dilemas y responsabilidades sociales de la profesión docente: Protagonismo docente en el campo educativo. En: Revista Proyecto Regional de Educación para América Latina y El Caribe -PRELAC, $n^{\circ} 1$. Santiago de Chile.

Sabalain, C. (2009). Introducción de conceptos básicos el medio ambiente y la estadística. Construcción de Indicadores Ambientales (ILAC/ODM/) en los países de América Latina. CEPAL.

Sánchez, O. (2011). Diseño de una propuesta sobre la implementación integrada de la educación ambiental en las instituciones educativas de Santiago de Cali. Cali: Universidad del valle instituto de educación y pedagogía Santiago de Cali.

Shymansky, J., Kyle, W. (1992). Stablishing a research agenda: critical issues of science curriculum reform. En: Revista Journal of Research in Science Teaching, vol. 29, $n^{\circ}$ 8.

Smith, N.J. (1997). ¿Qué es Educación Ambiental? Disponible en: http: //www. jmarcano.com/educa/njsmith.html, Recuperado en 24.08.2016

Torres, J. (1996). Globalización e interdisciplinariedad: el currículo integrado. España: Ed. Morata.

Ulloa, A. (2001). Transformaciones en las investigaciones antropológicas: sobre naturaleza, ecología y medio ambiente. En: Revista Colombiana de Antropología, vol. 37. Bogotá (Colombia)

Vasco, U. 1982. Documento de "Integración" en el diseño curricular, Universidad nacional de Colombia. Bogotá.

Vega, P., Álvarez, S. (2005). Planteamiento de un marco teórico de la Educación Ambiental para un desarrollo sostenible. En: Revista Electrónica de Enseñanza de las Ciencias, vol. $4, n^{\circ} 1$. España.

Vera, A. (2015). La huerta escolar como estrategia didáctica para el desarrollo de competencias científicas en la Institución Educativa Maestro Pedro Nel Gómez. Trabajo de investigación para optar al título de Maestría en Enseñanza de las Ciencias Exactas y Naturales. Facultad de Ciencias. Universidad Nacional -Sede Medellín. Colombia. 